PROCEEDINGS OF THE

AMERICAN MATHEMATICAL SOCIETY

Volume 132, Number 6, Pages 1861-1868

S 0002-9939(03)07261-7

Article electronically published on December 1, 2003

\title{
A GENERALIZATION OF A RESULT OF KAZHDAN AND LUSZTIG
}

\author{
JEFFREY D. ADLER AND STEPHEN DEBACKER
}

(Communicated by Rebecca Herb)

\begin{abstract}
Kazhdan and Lusztig showed that every topologically nilpotent, regular semisimple orbit in the Lie algebra of a simple, split group over the field $\mathbb{C}((t))$ is, in some sense, close to a regular nilpotent orbit. We generalize this result to a setting that includes most quasisplit $p$-adic groups.
\end{abstract}

\section{INTRODUCTION}

Suppose $G$ is the group of $\mathbb{C}((t))$-rational points of a simple, split, algebraic $\mathbb{C}((t))$ group. In [7, Corollary 4.1], Kazhdan and Lusztig show that if $Z$ is a topologically nilpotent, regular semisimple element of the Lie algebra of $G$ and $x$ is a special vertex in the Bruhat-Tits building of $G$, then there exists $g \in G$ such that the image of ${ }^{g} Z(=\operatorname{Ad}(g) Z)$ in the complex Lie algebra associated to $x$ is regular nilpotent. We generalize this result to a setting that includes most quasisplit groups over $p$-adic fields.

Motivation. Suppose $k$ is a $p$-adic field, and $G$ is the group of $k$-rational points of a connected reductive $k$-quasisplit group.

Our motivation for considering this problem came from harmonic analysis on $p$-adic groups; we were interested in what role the Bruhat-Tits building might play in stability questions. For example, we learned from lectures of Kottwitz that if $S$ denotes a Kostant section [9, §2.4] in $\mathfrak{g}$, then the map $G \times S \longrightarrow \mathfrak{g}$ given by $(g, Z) \longmapsto$ ${ }^{g} Z$ is a submersion. This implies that for a fixed regular nilpotent element $X$ in $\mathfrak{g}$ there is a neighborhood $U \subset \mathfrak{g}$ of $X$ with the following property: every stable regular semisimple orbit that intersects $U$ nontrivially contains a unique $G$-orbit that intersects $U$ nontrivially. The main result of this paper is the determination of a natural neighborhood of $X$ that has this property with respect to all regular semisimple topologically nilpotent elements.

Received by the editors September 23, 2002 and, in revised form, February 26, 2003

2000 Mathematics Subject Classification. Primary 22E35, 22E65; Secondary 17B45, 20G05, 20G15, 22E50, 20G25.

Key words and phrases. p-adic, Lie algebra, stability.

The authors were partially supported by the National Security Agency (\#MDA904-02-1-0020) and the National Science Foundation (Grant No. 0200542), respectively. This work was begun while the authors were attending workshops in Banff in 2001-2002 sponsored by the Mathematical Sciences Research Institute and the Pacific Institute for the Mathematical Sciences, and completed while the authors were visiting the Institute for Mathematical Sciences (IMS) at the National University of Singapore (NUS) in 2002, visits supported by IMS and NUS.

(C)2003 American Mathematical Society 
Acknowledgments. We heartily thank Gopal Prasad for many helpful comments and corrections. We thank George McNinch for several helpful conversations.

This paper. Let $k$ denote a complete field with nontrivial discrete valuation and residue field $\mathfrak{f}$. We suppose that $\mathfrak{f}$ is perfect. All extensions of $k$ that we consider will lie in a fixed algebraic closure $\bar{k}$ of $k$. Let $K$ denote the maximal unramified extension of $k$.

Let $\mathbf{G}$ denote a connected, reductive group with Lie algebra $\mathfrak{g}$. Let $G=\mathbf{G}(k)$ and $\mathfrak{g}=\mathfrak{g}(k)$. Let $\mathfrak{g}^{\text {r.s.s. }}$ denote the set of regular, semisimple elements in $\mathfrak{g}$. For any algebraic extension $E / k$ of finite ramification index, and any facet $F$ in the Bruhat-Tits building $\mathcal{B}(\mathbf{G}, E)$ of $\mathbf{G}(E)$, one can define a parahoric subgroup $\mathbf{G}(E)_{F}$ of $\mathbf{G}(E)$ [5]. Let $\mathbf{G}(E)_{F}^{+}$denote the pro-unipotent radical of $\mathbf{G}(E)_{F}$. As in [2], we define lattices $\mathfrak{g}(E)_{F}$ and $\mathfrak{g}(E)_{F}^{+}$in $\mathfrak{g}(E)$; for any $x \in \mathcal{B}(\mathbf{G}, E)$, we define $\mathfrak{g}(E)_{x}$ and $\mathfrak{g}(E)_{x}^{+}$to be $\mathfrak{g}(E)_{F}$ and $\mathfrak{g}(E)_{F}^{+}$, respectively, where $F$ is the unique facet in $\mathcal{B}(\mathbf{G}, E)$ containing $x$; and we define the $\mathbf{G}(E)$-invariant set $\mathfrak{g}(E)_{0^{+}}$to be $\bigcup_{F} \mathfrak{g}(E)_{F}^{+}$, where the union is taken over all facets $F$ in $\mathcal{B}(\mathbf{G}, E)$. When $E=k$, we denote the building by $\mathcal{B}(G)$, the reduced building by $\mathcal{B}^{\text {red }}(G)$, and the above objects by $G_{F}$, $G_{F}^{+}, \mathfrak{g}_{F}, \mathfrak{g}_{F}^{+}, \mathfrak{g}_{x}, \mathfrak{g}_{x}^{+}$, and $\mathfrak{g}_{0^{+}}$. This last object is the set of topologically nilpotent elements in $\mathfrak{g}$. We can identify $G_{F} / G_{F}^{+}$with the group of $\mathfrak{f}$-points of a connected reductive $\mathfrak{f}$-group $\mathrm{G}_{F}$. Let $\mathrm{L}_{F}:=\operatorname{Lie}\left(\mathrm{G}_{F}\right)$. We identify $\mathrm{L}_{F}(\mathfrak{f})$ with $\mathfrak{g}_{F} / \mathfrak{g}_{F}^{+}$.

Suppose $\mathbf{G}$ is $k$-quasisplit and let $X \in \mathfrak{g}$ be regular nilpotent (see 93 ). Suppose that we can complete $X$ to an $\mathfrak{s l}_{2}(k)$-triple $(Y, H, X)$. Under the hypotheses of Corollary 4.5 we can find a unique minimal facet $F \subset \mathcal{B}(G)$ such that the image of $F$ in $\mathcal{B}^{\text {red }}(G)$ is a special vertex and $Y, H, X \in \mathfrak{g}_{F}$. The main result of this paper is:

Proposition 1. Suppose that all of the hypotheses of 92 and of Corollary 4.5 are valid, that $\mathbf{G}, X$, and $F$ are as above, and that $Z \in \mathfrak{g}^{\text {r.s.s. }}$. Then $Z \in \mathfrak{g}_{0^{+}}$if and only if there is some $g \in \mathbf{G}(\bar{k})$ such that ${ }^{g} Z \in X+\mathfrak{g}_{F}^{+}$. Moreover, if $g^{\prime} \in \mathbf{G}(\bar{k})$ is such that ${ }^{g^{\prime}} Z \in X+\mathfrak{g}_{F}^{+}$, then ${ }^{g^{\prime}} Z={ }^{\ell g} Z$ for some $\ell \in G_{F}^{+}$.

In other words, the $\operatorname{coset} X+\mathfrak{g}_{F}^{+}$picks out a unique $G$-orbit in every topologically nilpotent, regular semisimple, stable orbit in $\mathfrak{g}$. Note that when $k=\mathbb{C}((t))$ and $\mathbf{G}$ is split and simple, we recover Corollary 4.1 of 7 .

To prove Proposition [ we will need some additional notation. Given a maximal $k$-split torus $\mathbf{S}$ of $\mathbf{G}$, we have the torus $S=\mathbf{S}(k)$ in $G$ and the corresponding apartment $\mathcal{A}(S)=\mathcal{A}(\mathbf{S}, k)$ in $\mathcal{B}(G)$. For any subgroup $\mathbf{H} \subset \mathbf{G}$, let $C_{\mathbf{G}}(\mathbf{H})$ denote the centralizer of $\mathbf{H}$ in $\mathbf{G}$. For $g \in \mathbf{G}$, let ${ }^{g} \mathbf{H}=g \mathbf{H} g^{-1}$. For $Y \in \mathfrak{g}$, we denote the centralizer of $Y$ in $\mathfrak{g}$ by $C_{\mathfrak{g}}(Y)$. For $Z \in \mathfrak{g}$, let $\mathbf{O}_{Z}$ denote the $\mathbf{G}$-orbit of $Z$ in $\mathfrak{g}$. Recall that for every algebraic extension $E / k$,

$$
\mathbf{O}_{Z}(E)=\mathbf{G}(\bar{k}) Z \cap \mathfrak{g}(E)=\bigsqcup_{Z^{\prime}} \mathbf{G}(E) Z^{\prime},
$$

where $Z^{\prime} \in \mathfrak{g}(E)$ ranges over the elements of $\mathbf{O}_{Z}(E)$ up to $\mathbf{G}(E)$-conjugacy.

\section{HYPOTHESES}

In this section, we list various properties which we require. Under some restrictions on $\mathbf{G}$ and $k$, all of these hypotheses are valid. In particular, they are all true when $\mathfrak{f}$ has characteristic zero. 
The first hypothesis is valid whenever the characteristic of $\mathfrak{f}$ is either zero or larger than some constant that can be determined by looking at the absolute root datum of $\mathbf{G}$. For more information, see [6].

Hypothesis 1. Let $X \in \mathfrak{g}$ be regular nilpotent. We can complete $X$ to an $\mathfrak{s l}_{2}(k)$ triple $(Y, H, X)$, produce a maximal $k$-split torus $\mathbf{S}$ in $\mathbf{G}$, and find a point $x \in$ $\mathcal{A}(\mathbf{S}, k)$, such that $H \in \operatorname{Lie}(\mathbf{S})(k), Y, H, X \in \mathfrak{g}_{x}$, and, for all finite extensions $E / k$,

$$
\mathbf{G}(E)_{x}^{+}\left(X+C_{\mathfrak{g}(E)_{x}^{+}}(Y)\right)=X+\mathfrak{g}(E)_{x}^{+} .
$$

When $G=\mathbf{S L}_{2}\left(\mathbb{Q}_{2}\right)$, this hypothesis fails, and so does Proposition 1 .

The remaining hypotheses are valid whenever $k$ has characteristic zero.

Hypothesis 2. Let $X$ and $\mathbf{S}$ be as in Hypothesis 1). Let $\mathbf{Z}=C_{\mathbf{G}}(\mathbf{S})$. (Since $\mathbf{G}$ is $k$-quasisplit, $\mathbf{Z}$ is a maximal $k$-torus.) For any algebraic extension $E / k$ over which $\mathbf{Z}$ splits, if $Z \in \operatorname{Lie}(\mathbf{Z})(E)$ is regular semisimple, then $X+Z$ is $\mathbf{G}(E)$-conjugate to $Z$.

Hypothesis 3. Suppose $Z \in \mathfrak{g}^{\text {r.s.s. }}$. For all $g \in \mathbf{G}(\bar{k})$ such that ${ }^{g} Z \in \mathfrak{g}(K)$, there exists $g^{\prime} \in \mathbf{G}(K)$ such that $g^{\prime} Z={ }^{g} Z$.

When the characteristic of $k$ is not a "torsion" prime for $\mathbf{G}$, then the centralizer of $Z$ in $\mathbf{G}$ is connected [15]. So this hypothesis follows immediately from Theorem III.2.3.1' of [11]. (See also Remark 1 in loc. cit.)

Hypothesis 4. Let $X$ and $Y$ be as in Hypothesis 1 . For any algebraic extension $E / k$, if $Z \in \mathfrak{g}(E)^{\text {r.s.s. }}$, then the set $\left(X+C_{\mathfrak{g}(E)}(Y)\right) \cap \mathbf{O}_{Z}(E)$ consists of one element.

This last hypothesis asserts the existence of a Kostant section (see, for example, [8, Theorem 0.10] and [9] $\S 2.4$ and $\S 4.3])$.

\section{Some notation And Results in a General Setting}

In this section only, let $k$ be any field.

General definitions. The term Levi subgroup will mean a rational Levi factor of a rational parabolic subgroup; a Levi subalgebra means the Lie algebra of a Levi subgroup.

If $L$ is a Levi subgroup of $G$, let $(L)$ denote the set of all subgroups of $G$ that are $G$-conjugate to $L$. If $M$ is another Levi subgroup of $G$, we write $(L) \leq(M)$ provided that ${ }^{g} L \subseteq M$ for some $g \in G$.

We call an element of the Lie algebra of a reductive group distinguished provided that it is nilpotent and does not lie in any proper Levi subalgebra. Similarly, an orbit in such a Lie algebra is said to be distinguished if some (hence any) element of it is distinguished.

For any $k$-group $\mathbf{H}$, let $\mathbf{X}_{*}(\mathbf{H})$ denote the set of one-parameter subgroups of $\mathbf{H}$, and let $\mathbf{X}_{*}^{k}(\mathbf{H})$ denote the subset of $k$-rational elements.

If $\mathbf{H}$ is connected and reductive, then $\lambda \in \mathbf{X}_{*}^{k}(\mathbf{H})$ determines a rational parabolic subgroup $\mathbf{P}_{\lambda}$ with rational Levi decomposition $\mathbf{P}_{\lambda}=\mathbf{M}_{\lambda} \mathbf{N}_{\lambda}$. Specifically, $\mathbf{P}_{\lambda}$ (resp. $\mathbf{M}_{\lambda}, \mathbf{N}_{\lambda}$ ) consists of those elements $g \in \mathbf{H}$ such that $\lim _{t \rightarrow 0}{ }^{\lambda(t)} g$ exists (resp. $=g,=1$ ). Note that $\mathbf{M}_{\lambda}=C_{\mathbf{H}}(\lambda)$, which is connected (by 13, Theorem 6.4.7]).

We will call an element $u \in \mathbf{H}(k)$ unipotent if there is some $\lambda \in \mathbf{X}_{*}^{k}(\mathbf{H})$ such that $\lim _{t \rightarrow 0}{ }^{\lambda(t)} u=1$. Similarly, we will call an element $X \in \operatorname{Lie}(\mathbf{H})(k)$ nilpotent if 
there is some $\lambda \in \mathbf{X}_{*}^{k}(\mathbf{H})$ such that $\lim _{t \rightarrow 0}{ }^{\lambda(t)} X=0$. The terms "unipotent" and "nilpotent" are sometimes given other definitions. See $\S 2.5$ of [2] for a discussion.

Comments on regular nilpotent elements. In this subsection, we discuss some results concerning regular nilpotent elements in $\mathfrak{g}$. Undoubtedly, these results are well known to the experts, but we could not find a reference.

Suppose $\mathbf{B} \subset \mathbf{G}$ is a rational Borel subgroup and $\mathbf{S} \subset \mathbf{G}$ is a maximal $k$-split torus contained in $\mathbf{B}$. Then we have associated sets $\Phi^{+}(\mathbf{S}, \mathbf{B}, \mathbf{G})$ and $\Delta(\mathbf{S}, \mathbf{B}, \mathbf{G})$ of positive and simple roots, respectively. Fixing an order on $\Phi^{+}(\mathbf{S}, \mathbf{B}, \mathbf{G})$, we may write each $u$ in the unipotent radical uniquely in the form $u=\prod_{\alpha \in \Phi^{+}(\mathbf{S}, \mathbf{B}, \mathbf{G})} u_{\alpha}$, where each $u_{\alpha}$ belongs to the root group corresponding to $\alpha$. For any such element $u, u_{\alpha}$ will always denote the factor of $u$ associated to $\alpha$. No statement that we make concerning $u_{\alpha}$ will depend on the ordering of the roots. Similarly, for any $X$ in the Lie algebra of the unipotent radical of $\mathbf{B}$, let $X_{\alpha}$ denote the projection of $X$ onto the $\alpha$-eigenspace in $\mathfrak{g}$.

Lemma 3.1. Suppose $\mathbf{G}$ is a $k$-quasisplit group and $u \in G$ is unipotent. If $u$ is regular, then there is a unique rational Borel subgroup $\mathbf{B} \subset \mathbf{G}$ such that $B=\mathbf{B}(k)$ contains $u$. Moreover, if $\mathbf{B}$ is a rational Borel subgroup such that $u \in B$, then $u$ is regular if and only if $u_{\alpha} \neq 1$ for all $\alpha \in \Delta(\mathbf{S}, \mathbf{B}, \mathbf{G})$, where $\mathbf{S}$ is any maximal $k$-split torus in $\mathbf{B}$.

Proof. Since $u$ belongs to the group of $k$-rational points of the derived group of $\mathbf{G}$, it is enough to assume that $\mathbf{G}$ is semisimple. From [14, Lemma 3.2 and Theorem 3.3], $u$ is regular if and only if it is contained in exactly one Borel subgroup $\mathbf{B}$ of $\mathbf{G}$.

Suppose $u$ is regular. Let $\mathbf{B}$ denote the unique Borel subgroup of $\mathbf{G}$ that contains $u$. By our definition of unipotent, $u$ is contained in the unipotent radical of some rational parabolic subgroup $\mathbf{P}$. Since $\mathbf{G}$ is $k$-quasisplit, there exists a rational Borel subgroup $\mathbf{B}^{\prime} \subset \mathbf{P}$ such that $u \in \mathbf{B}^{\prime}(k)$. By uniqueness, $\mathbf{B}=\mathbf{B}^{\prime}$.

We now consider the final statement of the lemma. Suppose that $\mathbf{B}$ is a rational Borel subgroup of $\mathbf{G}$ such that $u \in \mathbf{B}(k)$. Let $\mathbf{S}$ be a maximal $k$-split torus of $\mathbf{G}$ in $\mathbf{B}$ and let $\mathbf{T}=C_{\mathbf{G}}(\mathbf{S})$. If $\mathbf{U}$ denotes the unipotent radical of $\mathbf{B}$, then $\mathbf{B}=\mathbf{T} \mathbf{U}$ is a rational Levi factorization of $\mathbf{B}$ and $\mathbf{T}$ is a maximal $k$-torus in $\mathbf{G}$. From Lemma 3.2 of [14], $u_{\beta} \neq 1$ for all simple $\beta \in \Delta(\mathbf{T}, \mathbf{B}, \mathbf{G})$ if and only if $u$ is regular. Let $E$ be a Galois splitting field for $\mathbf{G}$ over $k$. Then each $\alpha \in \Delta(\mathbf{S}, \mathbf{B}, \mathbf{G})$ corresponds to a $\operatorname{Gal}(E / k)$-orbit in $\Delta(\mathbf{T}, \mathbf{B}, \mathbf{G})$. Thus, $u$ is regular if and only if $u_{\alpha} \neq 1$ for all $\alpha \in \Delta(\mathbf{S}, \mathbf{B}, \mathbf{G})$.

To transfer the above result to the Lie algebra, we will need to assume the following hypothesis.

Hypothesis E. There is a G-equivariant $k$-isomorphism from the unipotent variety in $\mathbf{G}$ to the nilpotent variety in $\mathfrak{g}$.

It follows from work of Springer [12] that Hypothesis E holds under certain mild restrictions on $\mathbf{G}$ and $k$.

Corollary 3.2. Suppose $\mathbf{G}$ is a k-quasisplit group, $X \in \mathfrak{g}$ is nilpotent, and Hypothesis $E$ is true for $\mathbf{G}$ and $k$. If $X$ is regular, then there is a unique rational Borel subgroup $\mathbf{B} \subset \mathbf{G}$ such that the nilradical of the Lie algebra of $B=\mathbf{B}(k)$ contains $X$. Moreover, if $\mathbf{B}$ is a rational Borel subgroup such that the nilradical of the Lie algebra of $\mathbf{B}$ contains $X$, then $X$ is regular if and only if $X_{\alpha} \neq 0$ for all $\alpha \in \Delta(\mathbf{S}, \mathbf{B}, \mathbf{G})$, where $\mathbf{S}$ is any maximal $k$-split torus in $\mathbf{B}$. 
Corollary 3.3. Suppose $X$ is a regular nilpotent element in $\mathfrak{g}$ and $\lambda \in \mathbf{X}_{*}^{k}(\mathbf{G})$ is a one-parameter subgroup such that $\lim _{t \rightarrow 0}{ }^{\lambda(t)} X=0$. If Hypothesis $E$ is true for $\mathbf{G}$ and $k$, then $\lambda \in \mathbf{X}_{*}^{k}(\mathbf{B})$, where $\mathbf{B}$ is the unique rational Borel subgroup containing $X$ in its Lie algebra. In particular, $C_{\mathbf{G}}(\lambda)$ is a maximal $k$-torus.

Proof. As usual, $\lambda$ determines a rational parabolic subgroup $\mathbf{P}_{\lambda}$ with rational Levi decomposition $\mathbf{P}_{\lambda}=\mathbf{M}_{\lambda} \mathbf{N}_{\lambda}$, where $\mathbf{M}_{\lambda}=C_{\mathbf{G}}(\lambda)$. Since $\lim _{t \rightarrow 0}{ }^{\lambda(t)} X=0$, we must have $X \in \operatorname{Lie}\left(\mathbf{N}_{\lambda}\right)$. Since there is a unique rational Borel subgroup containing $X$ in its Lie algebra, it follows that $\mathbf{P}_{\lambda}$ is this Borel subgroup.

\section{Some COMments on the PARAmetrization of nilpotent orbits}

We now return to our assumption that $k$ is complete with respect to a nontrivial discrete valuation and has perfect residue field $\mathfrak{f}$.

Remark 4.1. The hypotheses of [6, $\S 4.2]$ are enough to guarantee that Hypothesis $\mathrm{E}$ holds for $\mathbf{G}$ and $k$ and also for $\mathbf{G}_{F}$ and $\mathfrak{f}$ for every facet $F$.

Some notation. Let

$$
I^{d}:=\left\{(F, e) \mid F \text { is a facet in } \mathcal{B}(G), \text { and } e \in \mathrm{L}_{F}(\mathfrak{f}) \text { is distinguished }\right\} .
$$

Under some hypotheses on $k$ and $\mathbf{G}$ (see [6, §4.2]), to each pair $(F, e) \in I^{d}$ we can associate a nilpotent orbit $\mathcal{O}(F, e)$ in $\mathfrak{g}$ such that $\mathcal{O}(F, e)$ is the unique nilpotent orbit of minimal dimension that intersects $e$ nontrivially [6, Lemma 5.3.3].

For any $\mathfrak{s l}_{2}(k)$-triple $(Y, H, X)$ in $\mathfrak{g}$, we have the set

$$
\mathcal{B}(Y, H, X):=\left\{x \in \mathcal{B}(G) \mid Y, H, X \in \mathfrak{g}_{x}\right\} .
$$

This set is closed, convex, nonempty, and a union of facets [6, §5.1].

Suppose $\mathbf{S}$ is a maximal $k$-split torus in $\mathbf{G}$. Following [10, we associate to any facet $F \subset \mathcal{A}(\mathbf{S}, k)$ the Levi subgroup $M(F, \mathbf{S})$ that is generated by $C_{\mathbf{G}}(\mathbf{S})(k)$ and the root groups $U_{\dot{\psi}}(k)$, where $\dot{\psi}$ is the gradient of an affine root $\psi$ of $\mathbf{G}$ with respect to $\mathbf{S}, k$, and a nontrivial discrete valuation of $k$ such that the restriction of $\psi$ to $F$ is constant. Since $(M(F, \mathbf{S}))$ does not depend on $\mathbf{S}$, we may write $\left(M_{F}\right)$ instead.

Nilpotent orbits and Levi subalgebras. In this subsection, we associate to $(F, e) \in I^{d}$ a unique conjugacy class (namely, $\left(M_{F}\right)$ ) of Levi subgroups that are minimal with respect to the property that $\mathcal{O}(F, e)$ intersects the Lie algebra of some (hence any) element of this class nontrivially (compare to $\S 5$ of [3]). This answers a question of D. Kazhdan.

Proposition 2. Suppose the hypotheses of [6, §4.2] hold. Suppose that $(F, e) \in I^{d}$ and that $L$ is a Levi subgroup of $G$. If $\mathcal{O}(F, e) \cap \operatorname{Lie}(L) \neq \emptyset$, then $\left(M_{F}\right) \leq(L)$. Moreover, for every maximal $k$-split torus $\mathbf{S}$ of $\mathbf{G}$ with $F \subset \mathcal{A}(\mathbf{S}, k)$, we have $\mathcal{O}(F, e) \cap \operatorname{Lie}(M(F, \mathbf{S})) \neq \emptyset$.

Proof. The last claim follows immediately from [6, Corollary 4.3 .2 and Lemma 5.3.3(2)].

Suppose $L$ is a Levi subgroup of $G$ for which $\mathcal{O}(F, e) \cap \operatorname{Lie}(L) \neq \emptyset$. From [6] Lemma 5.3.3], we can produce an $\mathfrak{s l}_{2}(k)$-triple $(Y, H, X)$ in $\mathfrak{g}$ such that $Y, H, X \in \mathfrak{g}_{F}$ and $X \in \mathcal{O}(F, e)$. From [6] §5.5], $F$ is a maximal facet in $\mathcal{B}(Y, H, X)$. Since $\mathcal{O}(F, e) \cap \operatorname{Lie}(L) \neq \emptyset$, without loss of generality, we assume that $Y, H, X \in \operatorname{Lie}(L)$.

From the last paragraph of the proof of Theorem 5.6.1 of [6], there exists $\left(F^{\prime}, e^{\prime}\right)$ in the analogue of $I^{d}$ for $L$ such that $Y, H, X \in \operatorname{Lie}(L)_{F^{\prime}}$ and $X \in \mathcal{O}\left(F^{\prime}, e^{\prime}\right)$. Note 
that $F^{\prime}$ is a facet in $\mathcal{B}(L)$. If $F^{\prime \prime}$ is maximal among those facets of $\mathcal{B}(G)$ that lie in $F^{\prime}$, then $\left(M_{F^{\prime \prime}}\right) \leq(L)$.

On the other hand, $F^{\prime \prime} \subset \mathcal{B}(Y, H, X)$ and $F$ is a maximal facet in $\mathcal{B}(Y, H, X)$; so $\left(M_{F}\right) \leq\left(M_{F^{\prime \prime}}\right)$.

Remark 4.2. With suitable changes, the above result remains valid in the context of generalized $r$-facets.

\section{Some consequences.}

Corollary 4.3. Suppose the hypotheses of [6, $\S 4.2]$ hold. Suppose $(F, e) \in I^{d}$. The orbit $\mathcal{O}(F, e)$ is distinguished if and only if $F$ is a minimal facet in $\mathcal{B}(G)$.

Corollary 4.4. Suppose the hypotheses of [6, $\S 4.2]$ hold. If $X \in \mathfrak{g}$ is a distinguished element and $(Y, H, X)$ is an $\mathfrak{s l}_{2}(k)$-triple completing $X$, then there exists a unique point $x \in \mathcal{B}^{\text {red }}(G)$ such that $Y, H, X \in \mathfrak{g}_{x}$. Moreover, $x$ is a vertex.

Proof. Let $F$ be a maximal facet in $\mathcal{B}(Y, H, X)$ and let $(f, h, e)$ denote the $\mathfrak{s l}_{2}(\mathfrak{f})$ triple in $\mathrm{L}_{F}(\mathfrak{f})$ that is the image of $(Y, H, X)$. From [6, §5.5], we have that $(F, e) \in$ $I^{d}$. From [6. Lemma 5.3.3(2)], $X \in \mathcal{O}(F, e)$. From Corollary 4.3, $F$ is a minimal facet in $\mathcal{B}(G)$.

Corollary 4.5. Suppose $\mathbf{G}$ is k-quasisplit and the hypotheses of [6, §4.2] hold. Suppose $(F, e) \in I^{d}$. The orbit $\mathcal{O}(F, e)$ is regular if and only if $e \in \mathrm{L}_{F}(\mathfrak{f})$ is regular and the image of $F$ in $\mathcal{B}^{\text {red }}(G)$ is a special vertex for which (a choice of) the simple $\mathfrak{f}$-roots of $\mathbf{G}_{F}$ may be naturally identified with (a choice of) the simple $k$-roots of $\mathbf{G}$.

Proof. Suppose $\mathcal{O}(F, e)$ is regular. Then $\mathcal{O}(F, e)$ is distinguished. From Corollary 4.3, we may assume that $F$ is a minimal facet in $\mathcal{B}(G)$. Let $(f, h, e)$ be an $\mathfrak{s l}_{2}(\mathfrak{f})$ triple completing $e$, and let $(Y, H, X)$ be an $\mathfrak{s l}_{2}(k)$-triple lifting $(f, h, e)$ (see [6] 5.3.3(1)]). From [6, 5.3.3(2)], $X \in \mathcal{O}(F, e)$. Let $\lambda$ be the one-parameter subgroup adapted (see Definition 4.5.6 of [6]) to $(Y, H, X)$. Let $\mathbf{M}=C_{\mathbf{G}}(\lambda)$, and $M=\mathbf{M}(k)$. From [6, Corollary 4.5.9] we have $F \subset \mathcal{B}(M)$. From Corollary 3.2 Corollary 3.3 and Remark $4.1 \times$ lies in the Lie algebra of a unique rational Borel subgroup $\mathbf{B}$ of $\mathbf{G}$, and $\mathbf{M}$, a Levi factor of $\mathbf{B}$, is a maximal $k$-torus of $\mathbf{G}$. Let $\mathbf{B}$ denote the Borel $\mathfrak{f}$-subgroup of $\mathrm{G}_{F}$ corresponding to the image of $\mathbf{B}(k) \cap \mathrm{G}_{F}$ in $\mathrm{G}_{F}(\mathfrak{f})$. Note that $e$ belongs to the Lie algebra of $\mathrm{B}(\mathfrak{f})$.

Let $\mathbf{S}$ denote the maximal $k$-split torus in $\mathbf{M}$ and let $\mathbf{S}$ denote the corresponding maximal $\mathfrak{f}$-split torus in $\mathrm{G}_{F}$. Let $\mathfrak{g}(2)$ denote the 2-eigenspace for the action of $\lambda$ on $\mathfrak{g}$. From [6] Corollary 4.3.2 and Lemma 5.3.3(2)], $e \cap \mathfrak{g}(2) \subset \mathcal{O}(F, e)$. Hence, any element of $e \cap \mathfrak{g}(2)=X+\left(\mathfrak{g}_{F}^{+} \cap \mathfrak{g}(2)\right) \subset \operatorname{Lie}(\mathbf{B})(k)$ is regular. Thus, from Corollary 3.2 we must have that for all $Z \in e \cap \mathfrak{g}(2)$ and for all $\alpha \in \Delta(\mathbf{S}, \mathbf{B}, \mathbf{G}), Z_{\alpha} \neq 0$. This implies that every such $\alpha$, considered as a character of $\mathrm{S}$, must be a root in $\mathrm{G}_{F}$. Thus, we have an embedding of $\Delta(\mathbf{S}, \mathbf{B}, \mathbf{G})$ into $\Phi^{+}\left(\mathrm{S}, \mathrm{B}, \mathrm{G}_{F}\right)$, and by comparing dimensions we see that $\Delta(\mathbf{S}, \mathbf{B}, \mathbf{G})$ can be identified with $\Delta\left(\mathrm{S}, \mathrm{B}, \mathrm{G}_{F}\right)$. In particular, the image of $F$ in $\mathcal{B}^{\text {red }}(G)$ is special. Thus, $e_{\alpha} \neq 0$ for all $\alpha \in \Delta\left(\mathrm{S}, \mathrm{B}, \mathrm{G}_{F}\right)$; so $e$ is regular.

To prove the converse, suppose that the image of $F$ in $\mathcal{B}^{\text {red }}(G)$ is a special vertex, and $e \in \mathrm{L}_{F}(\mathfrak{f})$ is regular. Let $(e, h, f),(Y, H, X), \lambda$, and $\mathbf{M}$ be as above. From Corollary 4.3, $X$ is distinguished. Pick a maximal $k$-torus $\mathbf{T} \subset \mathbf{M}$ so that $\mathbf{T}$ contains a maximal $k$-split torus $\mathbf{S}$ with $F \subset \mathcal{A}(S) \subset \mathcal{B}(M)$. Since $\mathbf{T} \subset \mathbf{M}$, we have $\lambda \in \mathbf{X}_{*}^{k}(\mathbf{T})$. Thus, there exists a rational Borel subgroup $\mathbf{B}$ with Levi 
factorization $\mathbf{B}=\mathbf{T} \mathbf{U}$ such that $X \in \operatorname{Lie}(\mathbf{U})$. Let $\mathrm{B}, \mathrm{S}$, and $\mathbf{U}$ denote the objects in $\mathrm{G}_{F}$ corresponding to $\mathbf{B}, \mathbf{S}$, and $\mathbf{U}$, respectively. Note that $e \in \operatorname{Lie}(\mathbf{U})(\mathfrak{f})$. Since for all $\alpha \in \Delta\left(\mathrm{S}, \mathrm{B}, \mathrm{G}_{F}\right)$ we have $e_{\alpha} \neq 0$, we have that for all $\alpha \in \Delta(\mathbf{S}, \mathbf{B}, \mathbf{G}), X_{\alpha} \neq 0$. Hence, $X$ is regular.

\section{Proof of Proposition 1}

Produce an $\mathfrak{s l}_{2}(k)$-triple $(Y, H, X)$ completing $X$, a maximal $k$-split torus $\mathbf{S}$, and a point $x \in \mathcal{A}(\mathbf{S}, k)$ as in Hypothesis 1 . From Corollary 4.5, the image of $x$ in $\mathcal{B}^{\text {red }}(G)$ is special. Let $\mathbf{Z}$ denote the maximal $k$-split torus $C_{\mathbf{G}}(\mathbf{S})$. Fix $Z \in \mathfrak{g}^{\text {r.s.s. }}$. From Hypotheses 1 and 4 , it is enough to prove that

$$
Z \in \mathfrak{g}_{0^{+}} \Longleftrightarrow \mathbf{O}_{Z}(k) \cap\left(X+C_{\mathfrak{g}_{x}^{+}}(Y)\right) \neq \emptyset .
$$

"£": Suppose there exists $g \in \mathbf{G}(\bar{k})$ such that ${ }^{g} Z \in X+C_{\mathfrak{g}_{x}^{+}}(Y)$. (From [2] Corollary 3.2.6], this latter set is contained in $\mathfrak{g}_{0^{+}}$.) From Hypothesis 3, we may assume $g \in \mathbf{G}(K)$. From [1, Lemma 2.2.5], $\mathfrak{g}_{0^{+}}=\left(\mathfrak{g}(K)_{0^{+}}\right)^{\mathrm{Gal}(K / k)}$, the set of $\operatorname{Gal}(K / k)$-fixed points in $\mathfrak{g}(K)_{0^{+}}$. Therefore, $Z \in\left({ }^{g^{-1}} \mathfrak{g}(K)_{0^{+}}\right)^{\mathrm{Gal}(K / k)}=\mathfrak{g}_{0^{+}}$.

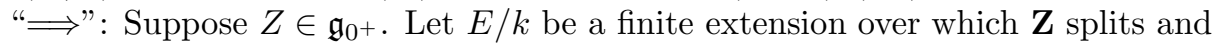
for which there exists $g \in \mathbf{G}(E)$ such that ${ }^{g} Z \in \operatorname{Lie}(\mathbf{Z})(E)$. Since $Z \in \mathfrak{g}_{0^{+}}$, we must have that $Z \in \mathfrak{g}_{y}^{+}$for some $y \in \mathcal{B}(G)$. Thus, $Z \in \mathfrak{g}(E)_{y}^{+} \subset \mathfrak{g}(E)_{0^{+}}$; so ${ }^{g} Z \in \mathfrak{g}(E)_{0^{+}}$. From [2] Theorem 3.1.2(2)] or [16, Lemma 8.2], ${ }^{g} Z \in \operatorname{Lie}(\mathbf{Z})(E)_{0^{+}} \subset \mathfrak{g}(E)_{x}^{+}$.

From Hypothesis 2 there exists $h \in \mathbf{G}(E)$ such that ${ }^{h g} Z={ }^{g} Z+X$. From Hypothesis 1 there exists $\ell \in \mathbf{G}(E)_{x}^{+}$such that ${ }^{\ell h g} Z \in X+C_{\mathfrak{g}(E)_{x}^{+}}(Y)$. Since $\mathbf{O}_{Z}(k)\left(\right.$ resp. $\left.\mathbf{O}_{Z}(E)\right)$ intersects $X+C_{\mathfrak{g}}(Y)\left(\right.$ resp. $\left.X+C_{\mathfrak{g}(E)}(Y)\right)$ exactly once (from Hypothesis (4), we conclude that ${ }^{\ell h g} Z \in X+C_{\mathfrak{g}_{x}^{+}}(Y) \subset \mathfrak{g}$. Therefore, $\mathbf{O}_{Z}(k) \cap(X+$ $\left.C_{\mathfrak{g}_{x}^{+}}(Y)\right) \neq \emptyset$.

\section{REFERENCES}

[1] J. D. Adler and S. DeBacker, Murnaghan-Kirillov theory for supercuspidal representations of tame general linear groups, submitted.

[2] J. D. Adler and S. DeBacker, Some applications of Bruhat-Tits theory to harmonic analysis on the Lie algebra of a reductive p-adic group, Michigan Math. J. 50 (2002), no. 2, 263-286. MR 2003g:22016

[3] P. Bala and R. W. Carter, Classes of unipotent elements in simple algebraic groups. II, Math. Proc. Cambridge Philos. Soc. 80 (1976), no. 1, 1-17. MR 54:5363b

[4] A. Borel, Linear algebraic groups, 2nd ed., Graduate Texts in Mathematics, vol. 126, SpringerVerlag, New York, 1991. MR 92d:20001

[5] F. Bruhat and J. Tits, Groupes réductifs sur un corps local I: Données radicielles valuées, Inst. Hautes Études Sci. Publ. Math. 41 (1972), 5-251. MR 48:6265

[6] S. DeBacker, Parametrizing nilpotent orbits via Bruhat-Tits theory, Ann. Math. 156 (2002), 295-332. MR 2003i:20086

[7] D. Kazhdan and G. Lusztig, Fixed point varieties on affine flag manifolds, Israel J. Math. 62 (1988), no. 2, 129-168. MR 89m:14025

[8] B. Kostant, Lie group representations on polynomial rings, Amer. J. Math. 85 (1963), 327404. MR 28:1252

[9] R. E. Kottwitz, Transfer factors for Lie algebras, Represent. Theory 3 (1999), 127-138 (electronic). MR 2000g:22028

[10] A. Moy and G. Prasad, Jacquet functors and unrefined minimal K-types, Comment. Math. Helvetici 71 (1996), 98-121. MR 97c:22021

[11] J.-P. Serre, Galois cohomology, Springer-Verlag, Berlin, 1997, Translated from the French by Patrick Ion and revised by the author. MR 98g:12007 
[12] T. A. Springer, The unipotent variety of a semi-simple group, Algebraic Geometry (Internat. Colloq., Tata Inst. Fund. Res., Bombay, 1968), Oxford Univ. Press, London, 1969, pp. 373391. MR 41:8429

[13] T. A. Springer, Linear algebraic groups, 2nd edition, Birkhäuser, Boston, 1998. MR 99h:20075

[14] R. Steinberg, Regular elements of semisimple algebraic groups, Inst. Hautes Études Sci. Publ. Math. 25 (1965), 49-80. MR 31:4788

[15] R. Steinberg, Torsion in reductive groups, Advances in Math. 15 (1975), 63-92. MR [50:7369

[16] J.-K. Yu, Construction of tame supercuspidal representations, J. Amer. Math. Soc. 14 (2001), no. 3, 579-622. MR 2002f:22033

Department of Theoretical and Applied Mathematics, The University of Akron, Akron, Ohio 44325-4002

E-mail address: adler@uakron.edu

Department of Mathematics, Harvard University, Cambridge, Massachusetts 02138

E-mail address: debacker@math.harvard.edu

Current address: Department of Mathematics, University of Michigan, Ann Arbor, Michigan 48109-1109

E-mail address: smdbackr@umich.edu 